\title{
Evaluating Discontinuities in Complex Systems: Toward Quantitative Measures of Resilience
}

\author{
Craig Stow $^{1}, \underline{\text { Craig R. Allen }}^{2}$, and $\underline{\text { Ahjond S. Garmestani }}^{3}$
}

\begin{abstract}
The textural discontinuity hypothesis (TDH) is based on the observation that animal body mass distributions exhibit discontinuities that may reflect the texture of the landscape available for exploitation. This idea has been extended to other complex systems, hinting that the identification and quantification of discontinuities in the distributions of appropriate variables may provide clues to emergent system properties such as resilience. We propose a discontinuity index, based on the vector norm of the full assemblage of observed discontinuities, as a means to quantify and compare this characteristic among systems. We also evaluate four methods to identify the number and location of the most prominent discontinuities. Although results of the four methods are similar, they are not identical, and we conclude that this problem is best addressed with a consistent operationally defined approach in an adaptive inference framework.
\end{abstract}

Key Words: discontinuities, textural discontinuity hypothesis, resilience, scalebreaks

\section{INTRODUCTION}

Holling (1992) proposed that animals have evolved physical and behavioral characteristics to exploit environmental texture at particular scales. Holling's textural discontinuity hypothesis (TDH) posits that animal body masses are entrained to the specific pattern and structure of available resources in a given system. Because resource distribution is discontinuous across landscape scales, animal body mass distributions should reflect this pattern and exhibit discontinuities consistent with the changes in the scale of resource availability. Holling viewed discontinuities in animal body mass distributions as indicative of evolutionary, self-organizing interactions of animals with the structures and processes specific to the scale at which they interact with their environment. Peterson et al. (1998) expanded this concept, postulating that ecosystem resilience is an emergent property resulting from functional redundancies across scales and functional diversity within scales.

Many subsequent studies have demonstrated results consistent with the TDH. For example, Raffaelli et al. (2000) found that experimentally altering marine sediment assemblages with size-specific perturbations of organic enrichment and predation caused densities and relative abundances of invertebrate taxa to shift, but there was little change in benthic biomass or the abundance size spectrum, maintaining a multimodal distribution of species. Havlicek and Carpenter (2001) compared body mass distributions in a set of experimental lakes, and reported that, despite changes in lake nutrient status and species composition, the multimodal body mass distributions of a wide range of species were conserved. Cumming and Havlicek (2002) used a cellular automaton model to evaluate body size distributions for 138 fish species. They found consistent modality in the data, and concluded that the structure detected was likely driven by ecological and evolutionary processes. Kamenir et al. (2004) analyzed seasonal and inter-annual variability in the size structure of phytoplankton of Lake Kinneret, Israel, and found that the assemblage was characterized by size classes separated by gaps. Stead et al. (2005) sampled a range of size fractions of stream benthic metazoans, and documented persistent changes in the number and locations of modes in the distribution, indicating that no single factor determines body size distributions. Stead et al. (2005) claim that breaks in body size distributions indicate an abrupt shift in the scale at 
which species operate in an environment; a pattern of peaks and troughs persists, regardless of the temporal and spatial variation in the data set. Others continue to document a relationship between discontinuities in body size distributions and habitat structure in freshwater fish (Fu et al. 2004) and nekton (Pittman et al. 2004). Szabo and Meszena (2006) used a resource use model to investigate the effect of natural selection on scale of perception. Their results indicate that discrete scales of opportunity act as distinct resources, and that species with similar perceptions are better at using resources at the same scale.

Accumulating evidence also supports the notion that discontinuities may be manifestations of scalespecific structuring processes that organize ecological systems, possibly with important management implications. For example, Allen et al. (1999) concluded that endangered and invasive species had body masses that fell near discontinuities - suggesting a possible tool to help forecast vulnerability or invasive potential. More recently, Scheffer and van Nes (2006) used a competition model to demonstrate that modality is an emergent property resulting from the coevolution of competitors. They contend that selforganized size classes represent scales of opportunity, separated by discontinuities, that are indicative of forbidden zones.

Thus, the analysis of the number and location of discontinuities in features from complex systems such as animal body mass distributions, or attributes representing other complex systems, such as city and firm size distributions (Garmestani et al. 2005, 2006), may provide insight into the structure, dynamics, and development of these systems. Additionally, if the pattern of discontinuities reflects differing scales of self-organizing processes, as these studies have suggested, then quantified measures of this attribute may provide a tool to assess resilience among ecosystems (Carpenter et al. 2005, Allen et al. 2005).

However, in addition to stirring considerable interest, Holling's proposal has evoked skepticism. Brown (1995) agreed that body mass distributions appear discontinuous, but offered an alternative explanation for the presence of a discontinuous pattern. Manly (1996) analyzed the TDH from the perspective of looking for body mass modes rather than discontinuities, and concluded that the body mass distributions examined were plausible small- sample outcomes from underlying unimodal or at most bimodal distributions. Siemann and Brown (1999) argued against the existence of discontinuities in mammal data sets by analyzing the gap size between modes, concluding that the observed gaps were not unusually large when compared with gaps generated from a continuous uniform distribution.

In this analysis, we consider the nature of the data on which the TDH is based, and evaluate approaches to identify and quantify the discontinuous structure in data from complex systems. These data include body mass information from several ecosystems, and firm and city size data from socioeconomic systems. We first evaluate the approaches offered by Manly (1996) and Siemann and Brown (1999) to provide perspective on the framework in which these data are currently viewed, and suggest alternatives.

\section{EXAMINING THE NUMBER OF MODES}

The TDH arose initially from the visual observation that adult animal body mass histograms appear discontinuously rather than smoothly distributed, and that this pattern occurs consistently in different systems (Holling 1992). Manly (1996) examined this supposition as a question of multimodality, asking: "Do the body mass distributions provide evidence for more than one mode?" Manly posed this question of the data sets originally examined by Holling using Silverman's (1981) kernel density method, and concluded that there was little evidence for more than two modes in any of the data examined.

Determining the number of modes in a distribution consisting of several underlying distributions is a difficult problem, particularly when the number and location of the component distributions is unknown. There is an extensive statistical literature on the subject exploring the properties of various methodologies. The problem becomes increasingly difficult as the number of suspected modes increases and the distance between modes decreases (Roeder 1994). Silverman's method is known to conservatively estimate the actual number of modes. In other words, in the test of the null hypothesis "the data likely arise from a distribution with one mode," Silverman's test will overestimate $P$ values, even in large samples (Hall and York 2001). Hall and York show in the simplest case, with an $\mathrm{H}_{\mathrm{A}}$ of two modes, the power of the test is low 
until modes become widely separated. Thus, the number of modes is likely to be underestimated using this method.

In addition, Manly (1999) clearly regarded his inquiry to be drawing inference about "the true underlying distributions" of the body mass data, implying that the available data are small samples from a large population. However, we argue that these data are more appropriately viewed as censuses, or near censuses, representing specific geographic regions. Most of the data considered have encompassed relatively large, easily observable species for which substantial undercounting is unlikely, thus the body mass distributions are more closely analogous to inventories than to samples. Although there is some measurement uncertainty in mean adult body mass estimates, measurement error usually obscures pattern, thus the presence of substantial error would not produce consistent patterns across systems. Therefore, the validity of the TDH is not appropriately evaluated by asking if the observed modes are "real" or just artifacts of sampling error, using a classical hypothesis testing approach.

\section{EXAMINING GAP SIZE}

Siemann and Brown (1999) approached this question differently, examining the size of the gaps (discontinuities) within body mass distributions, rather than the number of modes. They simulated adult body sizes using random values from uniform distributions tailored to conform to animal data sets from several biomes, and showed that the largest gap in most of the data sets examined was a plausible outcome from a continuous uniform process. In the few instances where the largest gap was unusually big, they sequentially evaluated the second and third largest gaps to show that there was not a succession of unusually big gaps. From this analysis, Siemann and Brown (1999) concluded that gaps were not a general feature of mammalian body mass distributions.

However, this testing does not accurately capture the premise of the TDH. The TDH is posited on the idea that adult body size distributions exhibit an assemblage of gaps, reflective of underlying processes, not that the gaps are unusually big. Small random samples from a uniform distribution will generally not be evenly distributed, they will exhibit gaps and modes (Fig 1). An evenly distributed sample from a continuous uniform distribution is unlikely (Fig. 1), more probably the result of a systematic than a random process. Thus, it is not clear that a uniform process provides an appropriate null hypothesis against which the TDH is appropriately tested.

Additionally, the method used by Siemann and Brown (1999) is more conservative than is immediately evident from the use of a $0.05 P$ value as a decision criterion, because this criterion was applied multiple times to each data set tested, increasing the likelihood that few body mass distributions would be found to have a set of unusually big gaps. They tested the second largest gap only if the largest gap was judged to be unusually big. Then, if the second largest gap also was judged unusually big, they tested the third largest gap, and so on. Assuming that the sizes of successive gaps are independent, the joint probability that the largest and second largest gaps will both be in the upper fifth percentile of their respective distributions is $0.05^{2}$, and the joint probability that the three largest gaps will all simultaneously be in the upper fifth percentile is $0.05^{3}$; thus, the test performed was actually much more stringent than it appeared.

However, this independence assumption invites investigation, as the size of the largest gap will influence the size of the successively smaller gaps. For example, if the largest gap is unusually small, then subsequent gaps will also tend to be small, because they must be smaller than the largest gap. Concurrently, if the largest gap is unusually big, it is less likely that subsequent gaps will also be big, because the gaps must sum to the total interval width spanned by the body masses. In the most extreme example, all organisms could conform to one of two body masses. In this case, the largest gap would equal the entire interval width, and all subsequent gaps would be of size zero, resulting in only one big gap.

Thus, there are conflicting constraints; unusually small largest gaps will tend to make subsequent gaps small, and unusually big largest gaps will also tend to make subsequent gaps small. To evaluate the net effect of these constraints on the independence assumption, we used a uniform random number generator to simulate animal body mass distributions with sample sizes ranging from $n=20$ to $n=100$. For each sample size, we generated 5000 outcomes from the uniform distribution, sorted the 
Fig. 1. Comparison of samples $(n=25)$ randomly generated from a uniform distribution (blue triangles) to systematically generated, evenly spaced samples (red triangles).

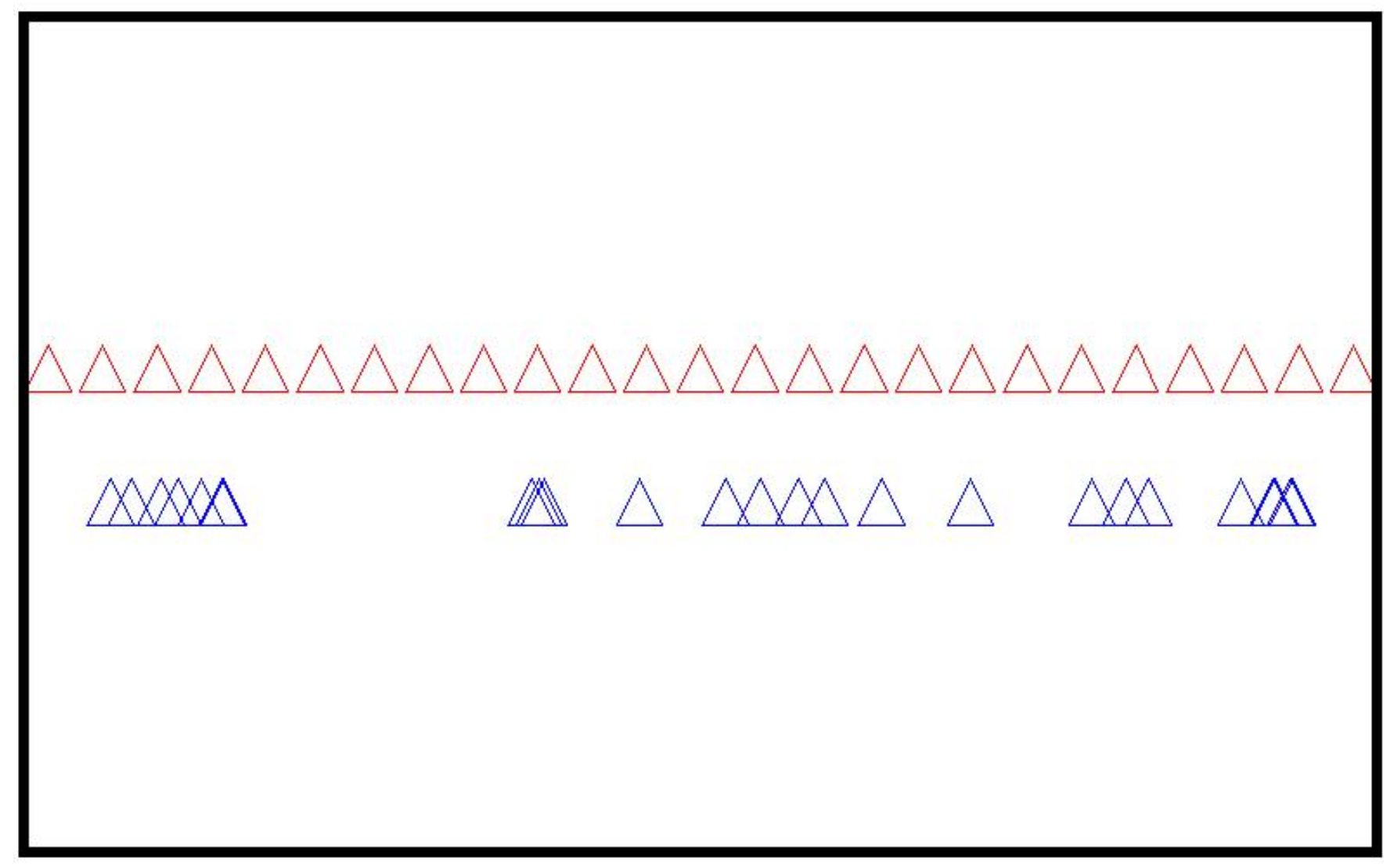

gaps by size, and tallied the proportion of the outcomes in which the first, second, and third largest gaps were in the upper $5^{\text {th }}$ percentile of their respective distributions.

The results (Table 1) confirm that the distributions of the largest, second largest, and third largest gaps are not independent. The constraint that successively smaller gaps must be smaller than their predecessors dominates the overall distribution structure, imposing a weak positive correlation (Fig. 2 ), and resulting in joint probabilities that are higher than would be expected if the distributions were independent (Table 1). However, these joint probabilities are still much lower than 5\%. Thus, we conclude that the testing performed by Siemann and
Brown (1999) was much less likely to find more than a few big gaps than was implied by a $0.05 P$ value.

\section{A DISCONTINUITY INDEX}

We argue that classical hypothesis testing is not an appropriate framework to evaluate the TDH; most data sets examined are near censuses rather than small samples, and there is no clear universally applicable null model. However, we propose that the uniform distribution does provide a basis to develop a discontinuity index (DI) useful to compare data sets, using the assemblage of 
Table 1. Comparison of the joint probability of the largest and second largest gaps, and the largest, second largest, and third largest gaps all simultaneously falling in the upper $5^{\text {th }}$ percentile of their respective marginal distributions. Results are based on 5000 random draws from uniform distributions for assemblages ranging from $n=20$ to $n=100$ observations.

\begin{tabular}{lll}
\hline \hline & $\begin{array}{l}\text { Proportion in upper } 5^{\text {th }} \text { percentile of } \\
\text { largest and } 2^{\text {nd }} \text { largest gap }\end{array}$ & $\begin{array}{l}\text { Proportion in upper } 5^{\text {th }} \text { percentile of } \\
\text { largest, } 2^{\text {nd }} \text { largest, and } 3^{\text {rd }} \text { largest gap }\end{array}$ \\
\hline Expectation if Independent $\rightarrow$ & 0.0025 & 0.000125 \\
$n$ & & \\
20 & 0.0044 & 0.0006 \\
30 & 0.0068 & 0.001 \\
40 & 0.007 & 0.0016 \\
50 & 0.0106 & 0.0024 \\
60 & 0.0112 & 0.0032 \\
70 & 0.0092 & 0.004 \\
80 & 0.0112 & 0.0034 \\
90 & 0.0098 & 0.0026 \\
100 & 0.0098 & 0.0044
\end{tabular}

discontinuities as a basis for comparison. The gap assemblage can be considered a vector, the magnitude or length of which is the vector norm (Friedberg and Insel 1986) calculated as:

$$
\sqrt{\sum_{i=1}^{m} g a p_{i}^{2}}
$$

where $\mathrm{m}$ is the number of gaps $(=n-1$, where $n$ is the number of species). Geometrically, this quantity is the higher dimensional analogue of calculating the length of the hypotenuse of a right triangle as the square root of the sum of the squared lengths of the other two sides. For a given interval, the largest possible value of this quantity occurs if all species lie on either end of the interval. In this case, there is one discontinuity that constitutes the entire interval width, and the rest of the gaps are all zero.
The smallest possible value of this index occurs if the species are evenly distributed, and all of the discontinuities are the same size. Therefore, a body mass distribution with similar-sized species, constituting only a few groups, will have a large vector norm, but if the body masses are evenly distributed the norm will be small.

We generated this index by taking 5000 draws from a $[0,1]$ uniform distribution, calculating the vector norm of the discontinuities for each of the 5000 draws, and calculating the cumulative density function (cdf) for each set of 5000 draws. We calculated and plotted the index for a range of plausible sample sizes, from $n=20-100$ by intervals of 10 (Fig. 3). Additionally, we calculated the vector norm for six example data sets to compare with the index. The data chosen represent four ecological examples: Boreal forest birds $(n=101)$ and mammals $(n=36)$ (Holling 1992), Everglades $(n=$ $35)$ and Mediterranean $(n=31)$ mammals (Allen et 
Fig. 2. Comparison of gap sizes generated from 5000 simulations. For each simulation, 50 numbers were randomly selected between 0 and 1, then the largest gap, second largest gap, and third largest gap were determined. $95^{\text {th }}$ percentiles are depicted by dotted lines-upper right quadrant is the upper $5^{\text {th }}$ percentile. A) Plot of largest gap vs. second largest gap, B) largest gap vs. third largest gap, C) second largest gap vs. third largest gap.

A.

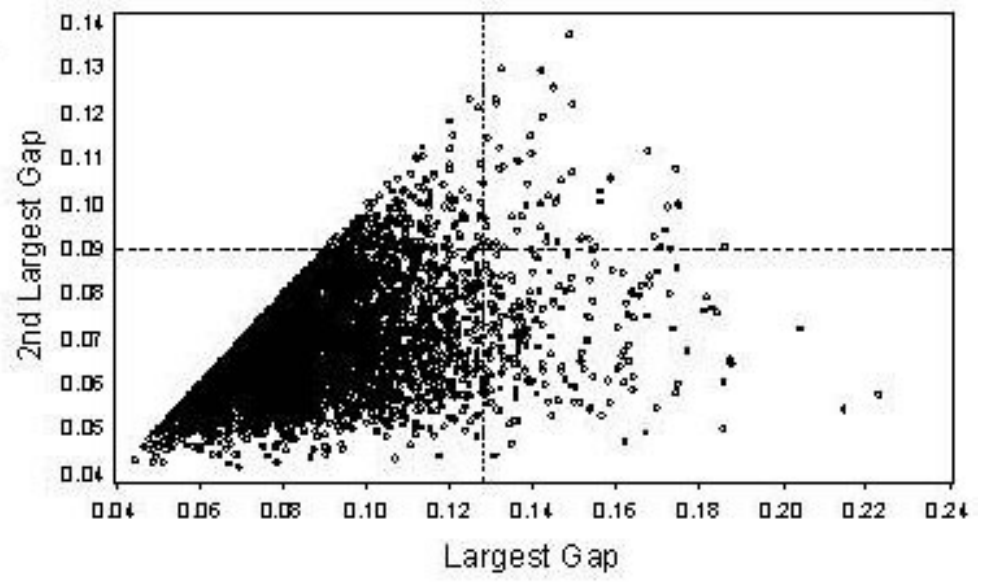

B.

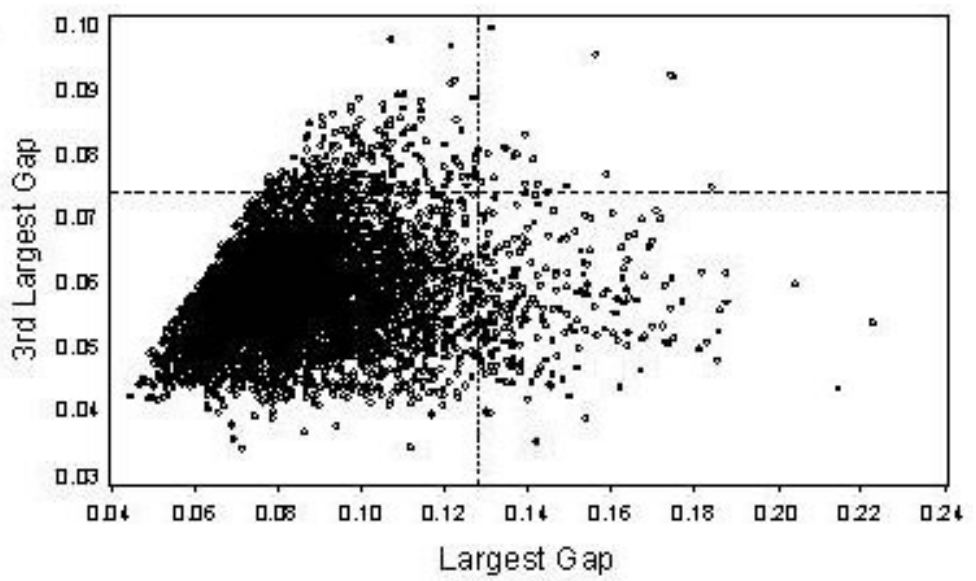

C.

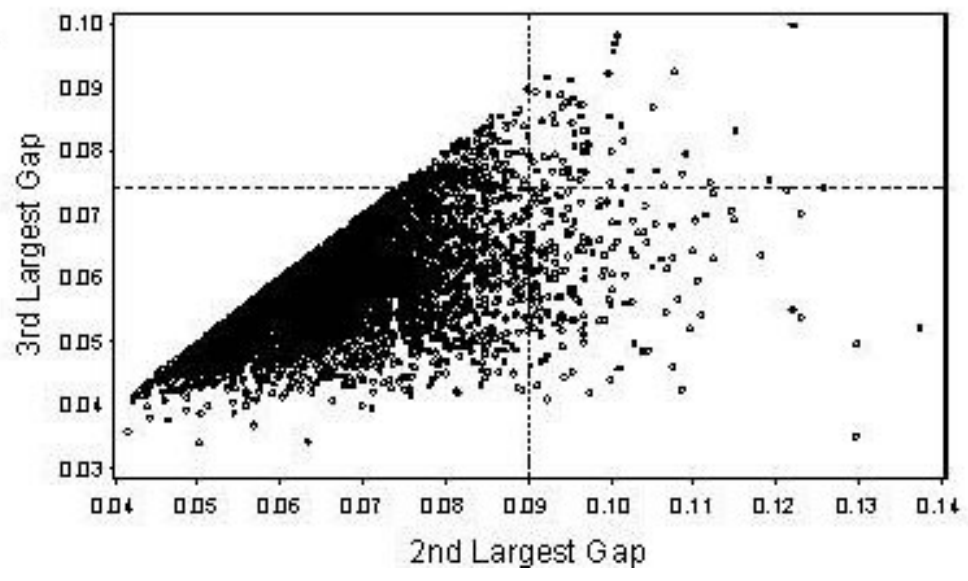


al. 1999), and two examples from other complex systems: regional city size data from the southwestern United States in $1890(n=48)$ (Garmestani et al. 2005), and regional manufacturing firm data (SIC Code 225) from South Carolina $(n=$ 63) (Garmestani et al. 2006). Each example data set was normalized to a $[0,1]$ interval by subtracting the value of the smallest observation from each entry then dividing each entry by the difference between the largest and smallest observation.

The six example data sets had a range of DI values. The 1890 SW US city data set had the highest DI, above the $99^{\text {th }}$ percentile for a data set of $n=48$. Boreal forest birds had a DI of 98.6, whereas SIC 226 firm data scored 95.8. Mediterranean mammals had the lowest DI at 46.0, and boreal forest and Everglades mammals were intermediate at 81.4 and 76.4 , respectively.

We suggest the vector norm of discontinuities as an index rather than a "significance test" because significance testing carries a fixed binary decision criterion-accept or reject. Instead, we propose this index as a useful tool to assist in the evaluation of structure in animal body mass (or other) distributions, providing a possible quantitative measure of resilience (Allen et al. 2005), and a basis for comparison among observed data sets.

\section{IDENTIFYING GROUPS}

Although this index provides a measure of "gappiness," there is still a need to develop rigorous approaches to evaluate where the discontinuities (and thus the groups) occur in a given data set (Allen et al. 2006). The structure and persistence of discontinuities has been proposed as a metric to assess resilience in complex systems (Allen et al. 2005), thus consistent approaches to determine this structure are necessary. Evaluating possible grouping in ordered data with $n$ observations is challenging, as there are $2^{n}-1$ possible ways to group the data.

Here we compare four candidate methods to determine groups, the "gap rarity index" (GRI) (Restrepo et al. 1997), hierarchical cluster analysis (HCA) (SAS Institute 1999), classification and regression trees (CART) (Breiman et al. 1984) and the Bayesian implementation of CART (BCART) (Chipman et al. 1998).
The GRI uses computer simulations that compare observed body mass distributions with a null distribution established by estimating a continuous unimodal kernel distribution of the log-transformed data (Silverman 1981). Gaps are defined as areas between successive body masses that significantly exceed the discontinuities generated by the continuous null distribution. A species aggregation is a grouping of three or more species with body masses not exceeding the expectation of the null distribution. This algorithm has been used previously in determining discontinuities and testing the TDH, but it is not readily available for broad application (Restrepo et al. 1997).

The HCA and CART algorithms work similarly, seeking to define homogenous groups in data, by successively splitting the data into two groups based on measures of within-group homogeneity. The resultant set of splits can be depicted as a branching tree, where the terminal nodes define groups of maximum homogeneity. However, a limitation of these methods is that the conditional recursive partitioning algorithm may result in a tree structure that is not globally optimal. A Bayesian implementation of the CART algorithm addresses this limitation by performing a stochastic search over the space of all possible trees, based on prior probabilities of a split occurring at any given node (Chipman et al. 1998). Selecting the best tree is based on the log integrated likelihood, a measure provided by the software. Considerable experimentation with this approach has indicated minimal sensitivity to the choice of prior probabilities, though the number of iterations required may be affected by poorly chosen priors. The Bayesian algorithm has been found to be particularly effective at detecting discontinuities in datasets (Bremner and Taplin 2004).

The four methods identified a similar number of discontinuities in the representative data sets, which were similar across methods, although they were never all identical (Table 2). The location of the discontinuities also was similar, resulting in interpretations that differ only slightly among methods (Fig. 4).

The methods described above provide results that contrast the conclusions of both Manly (1996) and Siemann and Brown (1999), and indicate that the distributions analyzed are discontinuously distributed. The differences in location and number of discontinuities confirm earlier suggestions that a 
Fig. 3. Discontinuity index based on vector norm of gap sizes. Solid lines (left to right) represent sample sizes of $n=160,120,80,60,40$, and 20. Dashed lines (left to right) represent example data sets with $n=$ 101 (boreal forest birds), $n=63$ (firm data SIC 226), $n=48$ (SW cities 1890), $n=36$ (boreal forest mammals), $n=35$ (Everglades mammals), and $n=31$ (Mediterranean mammals). Dots on dashed lines depict where the example data set vector norm is located on the cdf.

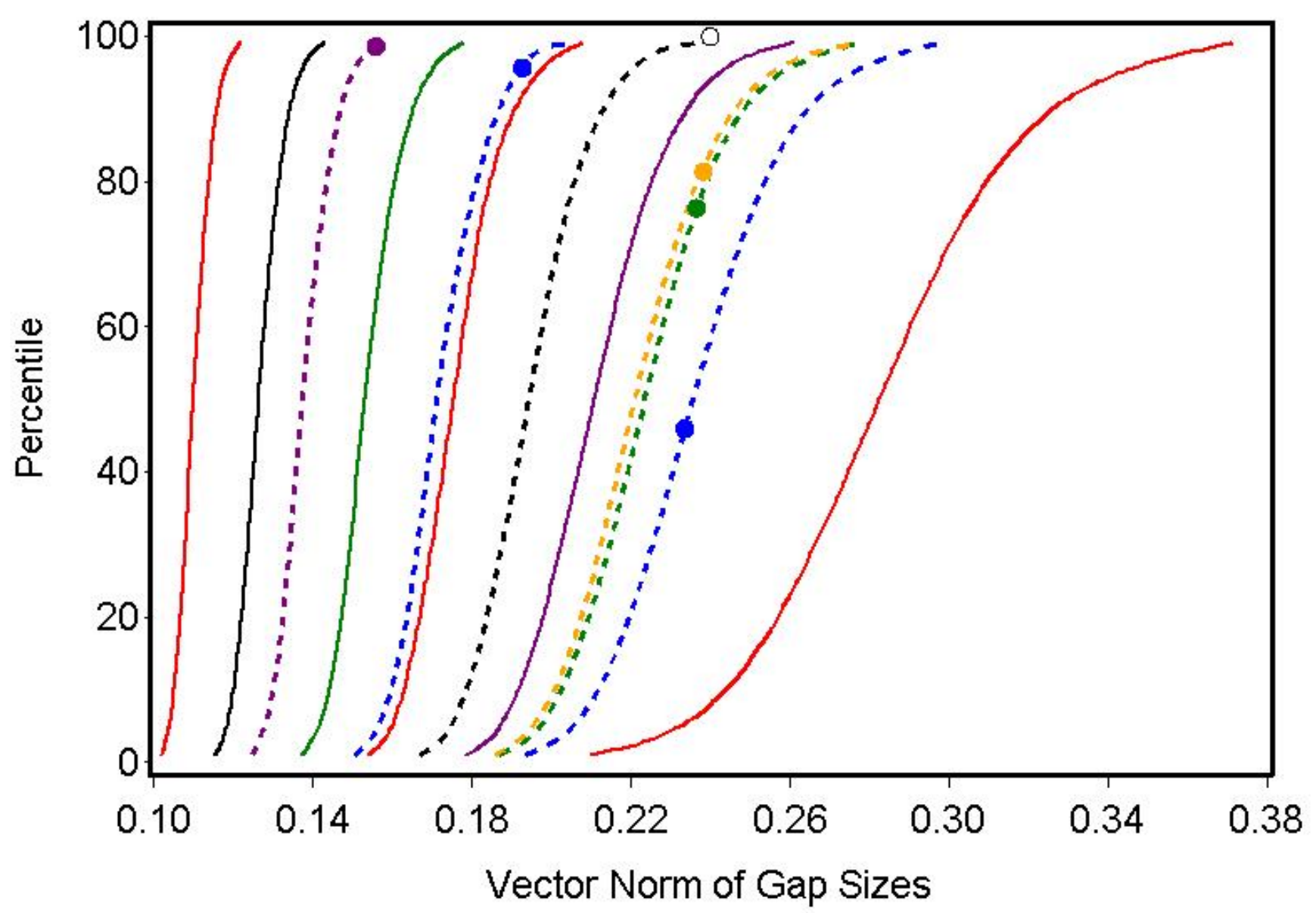

convergence of approaches should be used when interpreting results for discontinuities, given that none of the methods provides an unequivocal solution to the question "How discontinuous are these distributions and where are the discontinuities located?" Instead we recommend a consistent, operationally defined approach, using several methods in parallel.

We suggest the application of these quantitative methods in an adaptive inference framework (Holling and Allen 2002). Adaptive inference is appropriate for complex systems where structure derives from multiple sources operating at differing scales and where discrimination between pair-wise hypotheses is not possible. Adaptive inference uses a suite of tests that accumulates a body of evidence. Instead of pitting each member of a pair of hypotheses against each other, adaptive inference relies on exuberant invention of multiple, competing hypotheses, followed by an explanation of the logical consequences of each using carefully structured comparative data. It involves the accumulation of multiple lines of mutually reinforcing evidence that build a line of credible argument. Falsification, confirmation, deduction, 
Table 2. The number of discontinuities detected in six data sets by hierarchical cluster analysis (HCA), Bayesian classification and regression tree (BCART), classification and regression tree (CART), and gap rarity index (GRI).

\begin{tabular}{|c|c|c|c|c|}
\hline \multirow[t]{2}{*}{ Data set } & \multicolumn{4}{|c|}{ METHOD } \\
\hline & HCA & BCART & CART & GRI \\
\hline $\begin{array}{l}\text { Boreal forest birds }(n= \\
101)\end{array}$ & 8 & 8 & 7 & 8 \\
\hline $\begin{array}{l}\text { South Carolina } \\
\text { manufacturing firms } \\
\text { SIC } 226(n=63)\end{array}$ & 6 & 6 & 6 & 8 \\
\hline $\begin{array}{l}\text { Southwestern U.S. } \\
\text { cities } 1890(n=48)\end{array}$ & 3 & 4 & 4 & 4 \\
\hline $\begin{array}{l}\text { Boreal forest mammals } \\
(n=36)\end{array}$ & 5 & 5 & 5 & 4 \\
\hline $\begin{array}{l}\text { Everglades mammals } \\
(n=35)\end{array}$ & 5 & 5 & 6 & 5 \\
\hline $\begin{array}{l}\text { Mediterranean mammals } \\
(n=31)\end{array}$ & 4 & 4 & 5 & 4 \\
\hline
\end{tabular}

and induction are all employed to evaluate empirical evidence obtained by all manner of observations and tests for the purpose of sorting between hypotheses. Early stages of adaptive inference seek to minimize Type II error. Only later, as competing hypotheses have been sifted, empirical and experimental approaches seek to minimize Type I error. Adaptive inference is likely to facilitate methodological progress and understanding of the mechanisms responsible for the observed dynamics and structures in complex systems. Understanding the dynamics and structure of complex systems, such as ecosystems and economies, is a process. Identifying scales of structure in complex systems by identifying where discontinuities occur is an important step in that process.

\section{Acknowledgments:}

We wish to thank D. Otis and S. Carpenter for reviewing earlier versions of this manuscript. Support was provided by the James $S$. McDonnell Foundation 21 st Century Research Award/Studying Complex Systems (Allen). The Nebraska Cooperative Fish and Wildlife Research Unit is jointly supported by a cooperative agreement between the United States Geological Survey, the Nebraska Game and Parks Commission, the University of NebraskaLincoln, the United States Fish and Wildlife Service, and the Wildlife Management Institute. 
Fig. 4. Discontinuities detected by HCA, BCART, CART, and GRI approaches for boreal forest birds (top left), firm size SIC 226 (top right), SW cities 1890 (middle left), boreal forest mammals (middle right), Everglades mammals (bottom left), and Mediterranean mammals (bottom right). Dots at bottom represent data used in each analysis, dashed lines depict gaps (discontinuities).
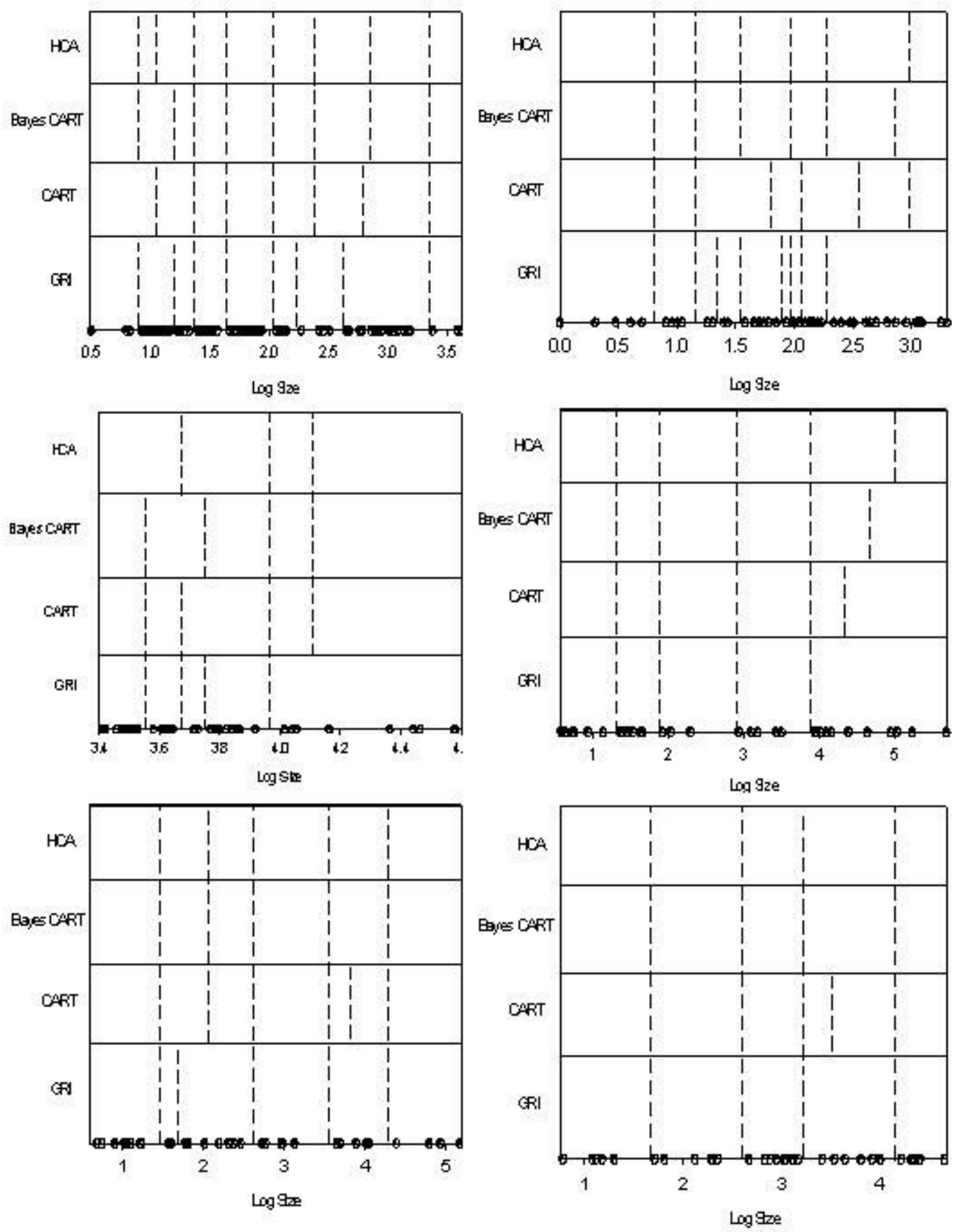


\section{LITERATURE CITED}

Allen, C. R., E. A. Forys, and C. S. Holling. 1999. Body mass patterns predict invasions and extinctions in transforming landscapes. Ecosystems 2:114-121.

Allen, C. R., A. S. Garmestani, T. D. Havlicek, P. A. Marquet, G. D. Peterson, C. Restrepo, C. A. Stow, and B. E. Weeks. 2006. Patterns in body mass distributions: sifting among alternative hypotheses. Ecology Letters 9:630-643.

Allen, C. R., L. Gunderson, and A. R. Johnson. 2005. The use of discontinuities and functional groups to assess relative resilience in complex systems. Ecosystems 8:958-966.

Allen, T. F. H., and T. B. Starr. 1982. Hierarchy: perspectives for ecological complexity. University of Chicago Press, Chicago, Illinois, USA.

Bremner, A. P., and R. H. Taplin. 2004. Performance of localized regression tree splitting criteria on data with discontinuities. Australian and New Zealand Journal of Statistics 46:367-381.

Breiman, L., J. H. Friedman, R. A. Olshen, and C. J. Stone. 1984. Classification and regression trees. Wadsworth International Group, Belmont, California, USA.

Brown, J. H. 1995. Macroecology. The University of Chicago Press, Chicago, Illinois, USA.

Brown, J. H., and P. F. Nicolleto. 1991. Spatial scaling of species composition - body masses of North American land mammals. American Naturalist 138:1478-1512.

Carpenter, S. R., F. Westley, and M. G. Turner. 2005. Surrogates for resilience of social-ecological systems. Ecosystems 8:941-944.

Chipman, H. A, E. I. George, and R. E. McCulloch. 1998. Bayesian CART model search. Journal of the American Statistical Association 93:935-948.

Cumming, G. S., and T. D. Havlicek. 2002. Evolution, ecology, and multimodal distributions of body size. Ecosystems 5:705-711.

Friedberg, S. H., and A. J. Insel. 1986.
Introduction to linear algebra with applications. Prentice-Hall, Englewood Cliffs, New Jersey, USA.

Fu, C., J. Wu, X. Wang, G. Lei, and J. Chen. 2004. Patterns of diversity, altitudinal range and body size among freshwater fishes in the Yangtze River basin, China. Global Ecology and Biogeography 13:543552.

Garmestani, A. S., C. R. Allen, and K. M. Bessey. 2005. Time series analysis of clusters in city size distributions. Urban Studies 42:1507-1515.

Garmestani, A. S., C. R. Allen, J. D. Mittelstaedt, C. A. Stow, and W. A. Ward. 2006. Firm size diversity, functional richness and resilience. Environment and Development Economics 11:533551.

Hall, P., and M. York. 2001. On the calibration of Silverman's test for multimodality. Statistica Sinica 11:515-536.

Havlicek, T., and S. R. Carpenter. 2001. Pelagic size distributions in lakes: are they discontinuous? Limnology and Oceanography 46:1021-1033.

Holling, C. S. 1992. Cross-scale morphology, geometry, and dynamics of ecosystems. Ecological Monographs 62:447-502.

Holling, C. S., and C. R. Allen. 2002. Adaptive inference for distinguishing credible from incredible patterns in nature. Ecosystems 5:319328.

Kamenir, Y., Z. Dubinsky and T. Zohary. 2004. Phytoplankton size structure stability in a mesoeutrophic subtropical lake. Hydrobiologia 520: 89104.

Manly, B. F. J. 1996. Are there clumps in body-size distributions? Ecology 77:81-86.

O'Neill, R. V., D. L. DeAngelis, J. B. Waide, and T. F. H. Allen. 1986. A hierarchical concept of ecosystems. Princeton University Press, Princeton, New Jersey, USA.

Peterson G., C. R. Allen, C. S. Holling. 1998. Ecological resilience, biodiversity, and scale. Ecosystems 1:6-18.

Pittman, S. J ., C. A. McAlpine, and K. M. 
Pittman. 2004. Linking fish and prawns to their environment: a hierarchical landscape approach. Marine Ecology Progress Series 283:233-254.

Raffaelli, D., S. Hall, C. Emes, and B. Manly. 2000. Constraints on body size distributions: an experimental approach using a small-scale system. Oecologica 122:389-398.

Restrepo C., L. M. Renjifo, and P. Marples. 1997. Frugivorous birds in fragmented neotropical montane forests: landscape pattern and body mass distribution. Pages 171-189 in W.F. Laurance and R.O. Bierregaard, editors. Tropical forest remnants: ecology, management and conservation of fragmented communities. University of Chicago Press, Chicago, Illinois, USA.

Roeder, K. 1994. A graphical technique for determining the number of components in a mixture of normals. Journal of the American Statistical Association 89:487-495.

SAS Institute, Inc. 1999. SAS user's guide: statistics, version 5 edition. Cary, North Carolina, USA.

Scheffer, M., and E. H. van Nes. 2006. Selforganized similarity, the evolutionary emergence of groups of similar species. Proceedings of the National Academy of Sciences 103:6230-6235.

Siemann, E., and J. H. Brown. 1999. Gaps in mammalian body size distributions reexamined. Ecology 80:2788-2792.

Silverman, B. W. 1981. Using kernel density estimates to investigate multimodality. Journal of the Royal Statistical Society B 43:97-99.

Stead, T. K., J. M. Schmid-Araya, P. E. Schmid, and A. G. Hildrew. The distribution of body size in a stream community: one system, many patterns. Journal of Animal Ecology 74:475-487.

Szabo, P., and G. Meszena. 2006. Spatial ecological hierarchies: coexistence on heterogeneous landscapes via scale niche diversification. Ecosystems 9:1009-1016. 